\title{
The value of antifungal therapy in allergic fungal rhinosinusitis*
}

S.G. Mistry ${ }^{1}$ and B.N. Kumar ${ }^{2}$

' ENT Registrar, Yorkshire \& the Humber Deanery, Bradford Royal Infirmary, Bradford, United Kingdom

2ENT Consultant, Royal Albert Edward Infirmary, Wigan, United Kingdom
Rhinology 52: 9-18, 2014

DOl:10.4193/Rhino13.100

*Received for publication:

July 12,2013

Accepted: September 2, 2013

\begin{abstract}
Background: Allergic fungal rhinosinusitis (AFS) is considered to part of the disease spectrum of chronic rhinosinusitis, which affects between five to fifteen per cent of the population. Currently, there is uncertainty relating to the pathological process and therefore optimal management of AFS. Studies assessing antifungal use have shown mixed results. The aim of this review is to assess the effect of antifungals on patients with AFS.
\end{abstract}

Methods: A systematic review of the literature to include all published trials searching Pubmed, Medline (Ovid), CINAHL (EBSCO) and the Cochrane central register of controlled trials (CENTRAL) databases.

Results: Sixteen studies (two systematic reviews, two meta-analysis, four randomised controlled trials, five prospective cohort studies and three retrospective studies) were included in this review. There was found to be no overall benefit of topical or oral antifungals upon endoscopic findings or patient reported outcome measures in AFS. There were no statistically significant differences in adverse effect profiles between treatment and control groups.

Conclusion: There is limited evidence to support the use of topical or oral antifungal agents in patients with AFS. Future research recommendations include large multicentre randomised trials with better matched patient groups and appropriate dosage and timing of antifungals.

Key words: rhinosinusitis, paranasal sinuses, antifungal agents, amphotericin b, endoscopy

\section{Introduction}

Rhinosinusitis in all of its forms (e.g. acute and chronic) is one of the most common conditions to affect the global population. There are estimated to be over 60 million sufferers within the European Union and the United States (US) alone ${ }^{(1)}$.

Allergic fungal rhinosinusitis (AFS) is considered to be a subtype of chronic rhinosinusitis (CRS) and it is estimated that approximately 5-10 per cent of those with CRS actually carry a diagnosis of AFS (2).

There is much debate and controversy regarding AFS and the possible role that fungi may play in CRS. Rather than being a distinct entity, it has been argued that AFS falls within the large spectrum of sino-nasal inflammatory disease which includes the presence of nasal polyps. A growing body of experts now believe that fungi act as a primary stimulus within CRS and have therefore proposed the term "eosinophilic fungal rhinosinusitis". The consensus upon the effective management of AFS involves firstly removing the fungal stimulus (often surgical), followed by control of the immunological response and recurrence ${ }^{(3)}$.

Antifungal therapy is believed to reduce the antigenic load and therefore reduce the hypersensitivity (allergic) response and recurrence. Several studies assessing systemic antifungal agents (e.g. Itraconazole and Ketaconazole) have shown mixed 
results with regards to disease control and recurrence within this patient group ${ }^{(4)}$. As AFS is a non-invasive condition, it is considered that topical antifungal therapy should be more effective and safer in providing effective local disease control. Several investigators have shown support for the use of topical antifungal agents in AFS ${ }^{(5,6)}$ as selected studies have reported some promising outcomes. However, there are also many studies that have shown no benefit. A previous Cochrane review (2011) has assessed the role of antifungals in CRS, however, there is little discussed with regards to the subgroup of AFS patients ${ }^{(7)}$. The major factor for this limited analysis was a lack of high quality evidence available in the literature regarding patients with AFS (did not fulfil inclusion criteria). Therefore, this review aims to assess the current evidence-base focusing on the use of antifungals in AFS.

\section{Materials and methods}

\section{Objectives}

To investigate the effect of antifungal therapy (topical and systemic) on the objective nasal endoscopic findings in patients with AFS.

\section{Searching the literature}

Types of studies

Following a review of the available literature a decision was made to include meta-analysis, systematic reviews, placebo controlled randomised control trials (RCT), open prospective studies and retrospective reviews within this study.

\section{Types of participants}

Studies that recruited both adults and children diagnosed with AFS as defined by the Bent and Kuhn (1994) criteria ${ }^{(8)}$ (or modifications to Bent and Kuhn criteria ${ }^{(8)}$ ) were included (Table 1). Also due to the similarity in the disease process, secondary analysis included adults and patients diagnosed with CRS and its subtypes (with and without nasal polyposis) as described by the European Position Paper on Rhinosinusitis and Nasal Polyps (2012) ${ }^{(9)}$, or by the American Academy of Otolaryngology - Head and Neck Surgery guidelines ${ }^{(10)}$.

\section{Types of interventions}

Studies involving the use of systemic and topical antifungals in patients with AFS were considered in the primary analysis. Systemic antifungals can be administered orally or intravenously. Topical antifungals can be administered via nasal inhalation, irrigation, drops, sprays and douching. Further analysis was performed on studies using systemic and topical antifungals in CRS and its sub-types.

\section{Outcome measures}

Objective endoscopic findings characterised by several endoscopic staging systems (e.g. Kupferberg staging system, modified Lund-Kennedy \& Malm) ${ }^{(11-13)}$ were used as the primary outcome measures. Secondary outcome measures included Patient Reported Outcome Measures (PROM), collated symptom scores and subjective symptom findings (e.g. improvement, no improvement or deterioration). Adverse events were also recorded.

\section{Data collection and analysis \\ Literature search}

An electronic database literature search was conducted with no date or language restrictions. This as well as other similar systematic searches are at risk of publication bias through non-inclusion of unpublished studies. A search strategy was performed with a combination of Medical Subject Headings (MeSH) and keyword items. Medical databases included Pubmed, Medline (Ovid) and CINAHL (EBSCO), the Cochrane central register of controlled trials (CENTRAL). Following initial search results, secondary hand-searches were performed. Search terms included "allergic fungal sinusitis", "fungal sinusitis", "sinusitis", "rhinosinusitis", "paranasal sinuses", "antifungal agents", "Amphotericin B", "Itraconazole", "Fluconazole", "Ketoconazole" and "Voriconazole”. The evidence was individually analysed according to critical appraisal selection programme (CASP) data collection tools as highlighted by the Centre for Evidence Based Medicine (2009) ${ }^{(14)}$. The methods and quality (to include outcomes) were evaluated in turn.

Particular consideration was given to:

$\begin{array}{ll}\text { - } & \text { Trial characteristics } \\ \text { - } & \text { Methods of randomisation } \\ \text { - } & \text { Intention to treat analysis } \\ \text { - } & \text { Duration of trial (to include follow-up) } \\ \text { - } & \text { Number of participants } \\ \text { - } & \text { Age of participants } \\ \text { - } & \text { Exclusion criteria } \\ \text { - } & \text { Diagnostic criteria } \\ \text { - } & \text { Interventions - primary } \\ \text { - } & \text { Interventions - secondary } \\ \text { - } & \text { Duration of treatment } \\ \text { - } & \text { Outcomes (primary and secondary) } \\ \text { - } & \text { Adverse outcomes }\end{array}$

Assessment for risk of bias from included randomised controlled studies

Critical Appraisal Skills Programme tools ${ }^{(14)}$ were used to evaluate sequence generation, allocation concealment, blinding of participants, outcome assessors, incomplete outcome data, selective outcome reporting and other sources of bias. 
Table 1. Bent and Kuhn Criteria (1994) for the diagnosis of allergic fungal sinusitis ${ }^{(8)}$.

\section{Bent and Kuhn Criteria}

Evidence of Gel \& Coombs type I hypersensitivity

Nasal polyposis

Characteristic Computer Tomography findings

Eosinophilic mucin

Positive Fungal Smear

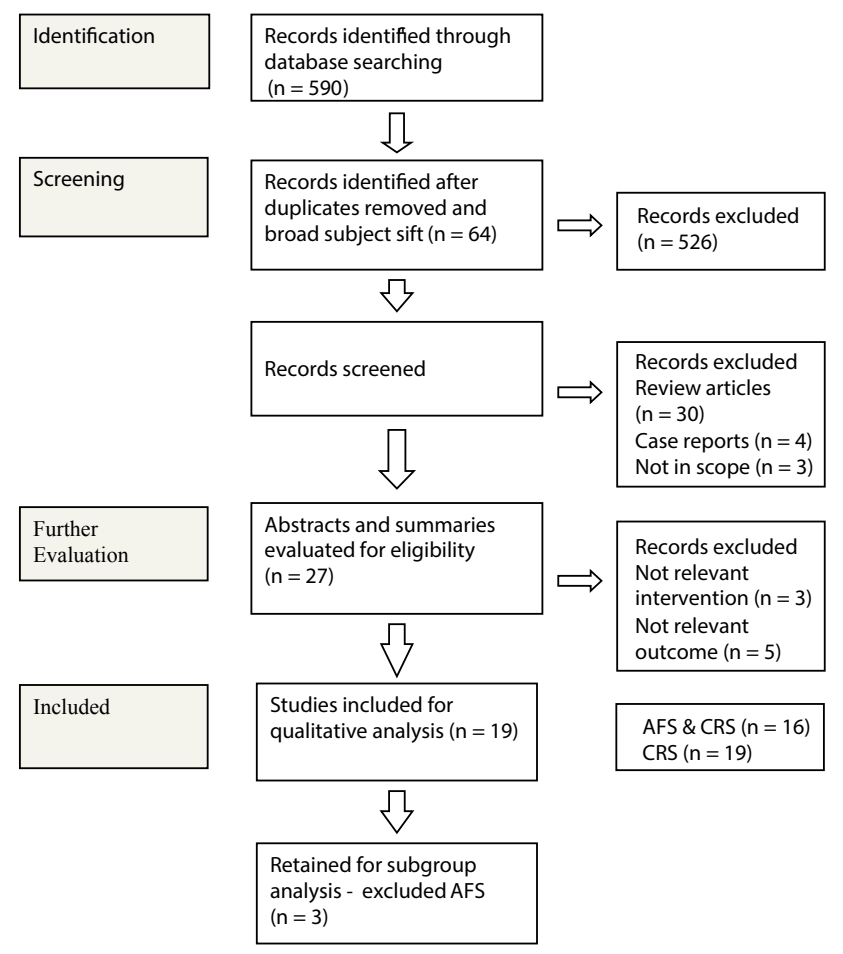

Figure 2. Flow diagram illustrating the literature selection process.

\section{Results}

\section{Description of studies}

\section{Results of the search}

A literature search revealed 590 references, 526 references were initially removed by a primary screening process, which involved identifying and excluding clearly irrelevant material and duplicates from searches. The remaining 64 references underwent a secondary screening process and assessment. Following review of abstracts and summaries, 19 studies were selected for review. From these, 16 included participants with AFS, the remainder were included as they investigated CRS and the subtypes. A flow chart that details the study selection process can be found in Figure 2.

\section{Methodological quality of included trials}

Detailed information regarding the characteristics of each trial included within this review can be found in Table 2 .

Overall, from the four RCTs included within this review, significant limitations in methodology leading to bias were identified from the study by Khalil et al. ${ }^{(15)}$ (Table 3).

\section{Exclusion of studies}

Twenty-seven trials were identified as appropriate to the basic criteria for the review. Eight of these trials did not focus upon antifungals within AFS or CRS and its subtypes. This left 19 trials. A full text article review revealed that three of these trials actively excluded AFS from its analysis. As the outcomes of these three studies were relevant to several of the secondary outcome measures related to this study (i.e. antifungals in CRS), they have been included as a further sub-group analysis as indirect conclusions can be drawn from these studies. Excluding these studies from the primary analysis, the final search identified 16 studies that assessed the use of antifungals in AFS and CRS.

\section{Outcome measures}

\section{Endoscopic outcomes - topical antifungals}

Data extracted from an existing meta-analysis has shown there to be no overall evidence for improvement in endoscopic scores following the use of topical or oral antifungals in AFS and CRS ${ }^{(7)}$. The two trials that included AFS patients ${ }^{(16,17)}$ interestingly marginally favoured the treatment groups (SMD -0.97 [-1.83 to -0.10 $95 \% \mathrm{Cl}^{(16)}$ and $\left.-0.13[-0.62 \text { to } 0.3695 \% \mathrm{Cl}]^{(17)}\right)$. However, although the overall meta-analysis reported no benefit, it must be noted that it included studies that actively excluded AFS (7). Ponikau et al. included patients with both AFS and CRS ${ }^{(17)}$. As mentioned, the group reported improved endoscopic outcomes ${ }^{(11)}$. However, the group only enrolled a small number of participants ( $\mathrm{n}$ $=30$, both CRS and AFS) and suggested that factors including a poor uptake in the study and a national shortage of Amphotericin $B(A M B)$ to be contributing factors for such low enrolment numbers. Although 20 per cent of participants did not complete the study, attrition bias is believed to be low as the group had calculated from previous studies that treatment effect was not expected until ten to twelve weeks, and as the participants withdrew prior to this they did not need to be included within the analysis.

Although the meta-analysis marginally favoured the treatment group in the study by Liang et al. ${ }^{(16)}$, the authors reported no significant benefit upon endoscopic outcome following use of topical AMB. This high quality study included both AFS and CRS participants $(n=70)$. Despite robust methodology, the study only assessed the effect of topical AMB over a four week dura- 


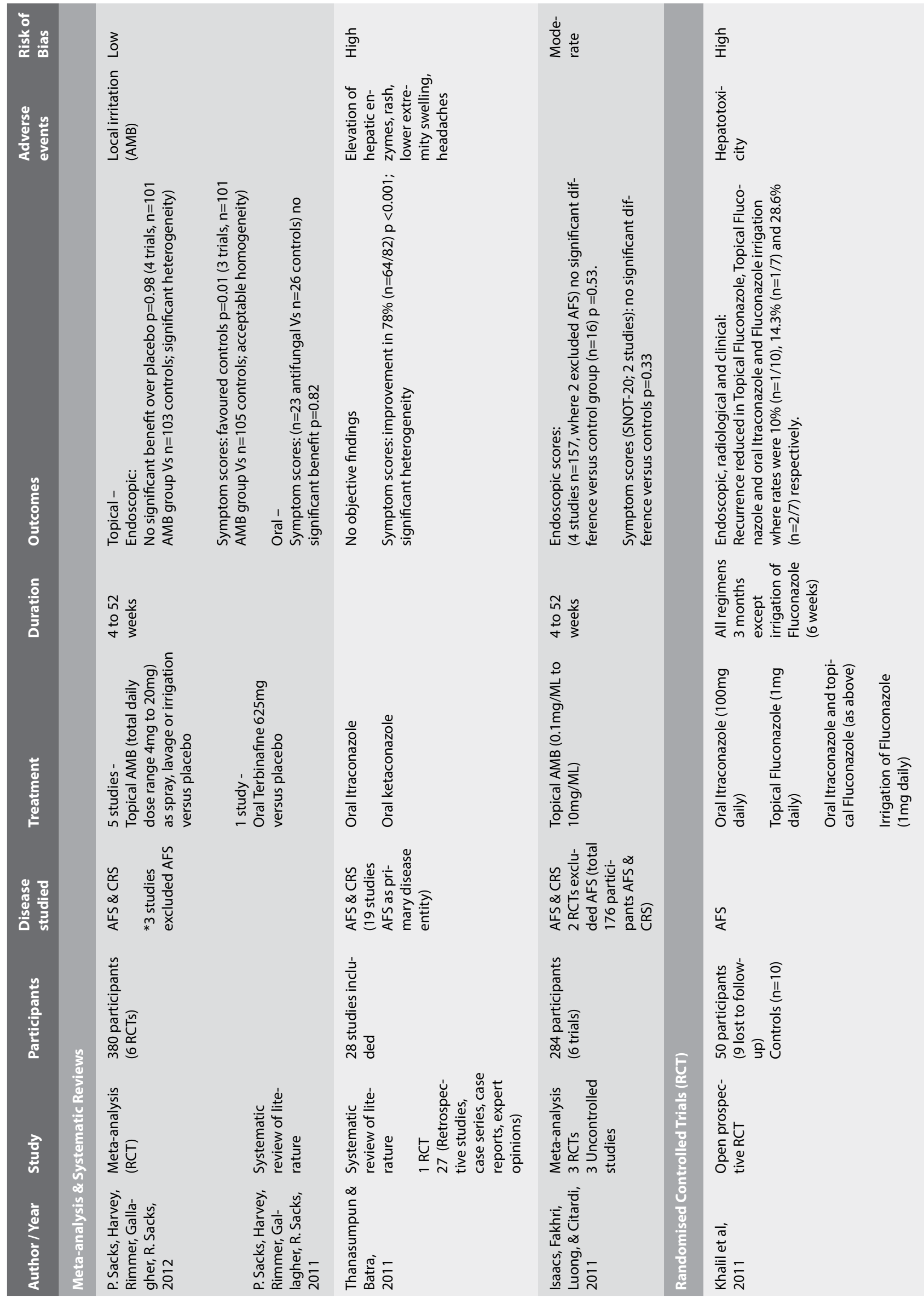


Table 2. Studies included in the review, continued...

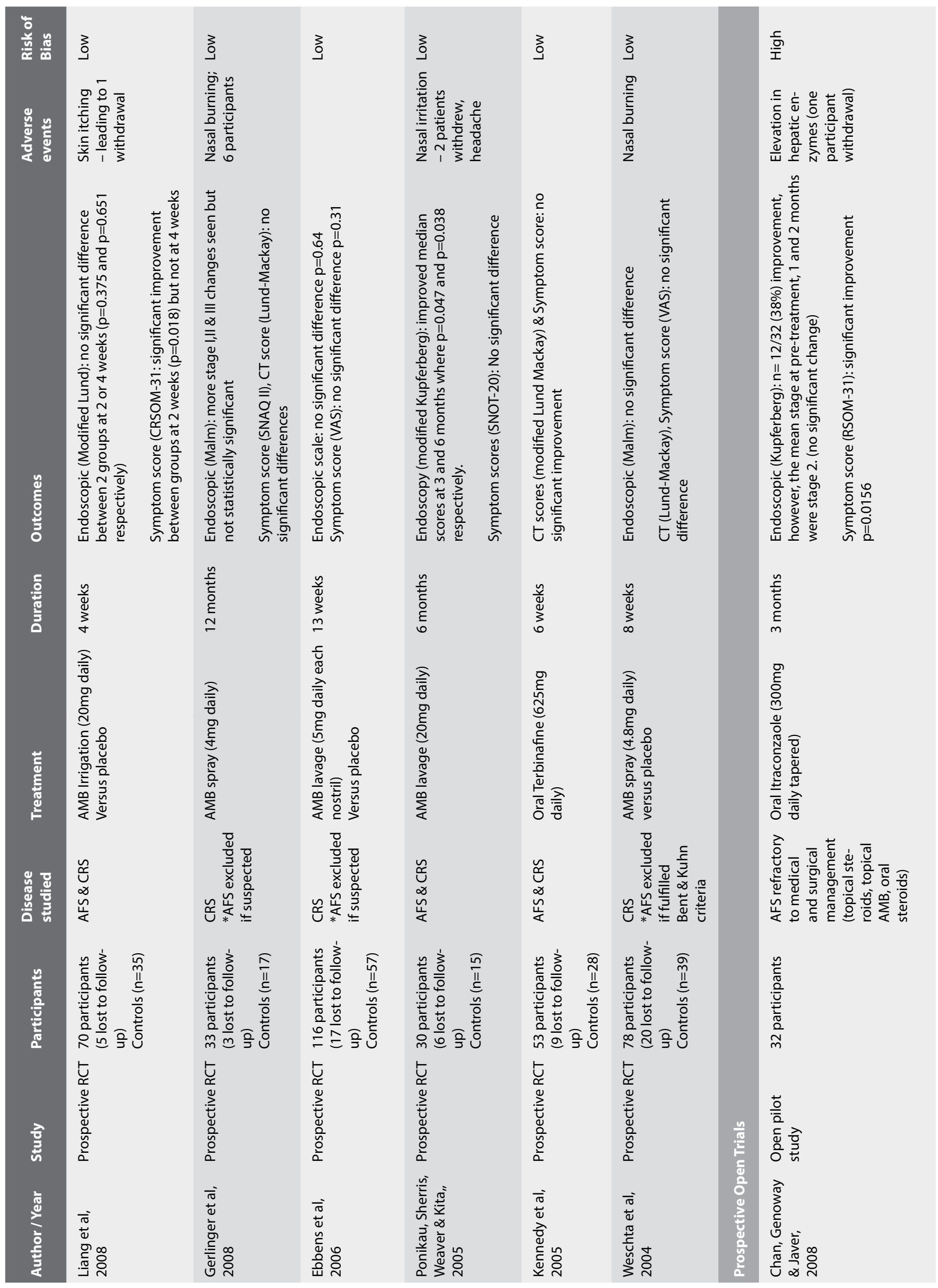


Table 2. Studies included in the review, continued...

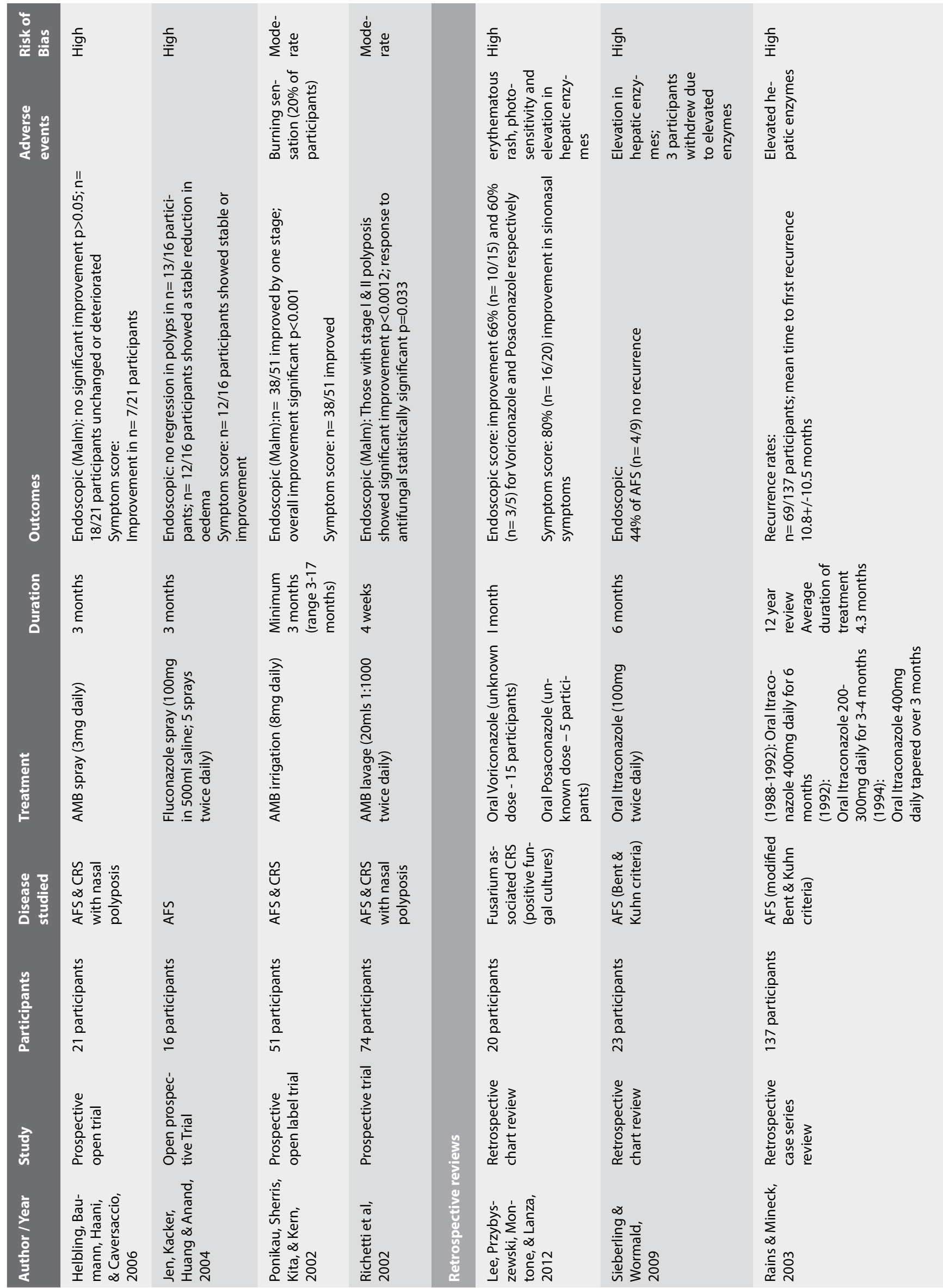


Table 3. Methodology of included randomised controlled trials.

\begin{tabular}{|lcccccccc} 
Study & $\begin{array}{c}\text { Randomisation } \\
\text { techniques }\end{array}$ & $\begin{array}{c}\text { Allocation con- } \\
\text { cealment }\end{array}$ & Blinding & $\begin{array}{c}\text { Incomplete } \\
\text { outcome data }\end{array}$ & $\begin{array}{c}\text { Selective out- } \\
\text { come reporting }\end{array}$ & $\begin{array}{c}\text { Other sources } \\
\text { of bias }\end{array}$ & $\begin{array}{c}\text { Risk of bias } \\
\text { (author judge- } \\
\text { ment) }\end{array}$ \\
\hline Kennedy et al., 2005 & Computerised & Unclear & Double & Accounted & No & Unclear risk & Low \\
Ponikau et al., 2005 & Block & Yes & Double & Accounted & No & Low risk & Low \\
Liang et al., 2008 & Block & Yes & Double & Accounted & No & Low risk & Low \\
Khalil et al., 2011 & Not described & No & No & Not accounted & Yes & Moderate risk & High
\end{tabular}

tion. It has been previously reported that this length of intervention is unlikely to yield meaningful outcomes ${ }^{(17)}$.

Khalil et al. exclusively assessed AFS participants and reported evidence of objective improvement and reduced recurrence rates when assessing similarly matched groups ${ }^{(15)}$. However, several significant limitations were noted from the study, which can be seen in Table 3. In addition to these, it was not clearly stated how the recurrence was measured endoscopically (e.g. objective score / technique). Evidence derived from this study must be used with great caution due to the numerous failings and bias identified.

Two open prospective trials ${ }^{(18,19)}$ reported improved endoscopic scores ${ }^{(13)}$ following their antifungal regimes. The two groups assessed participants with AFS and CRS with and without nasal polyposis. The heterogeneity of the patient groups (in terms of the disease stage) make results difficult to interpret. It was also reported that participant surgical state in the study by Richetti et al. influenced outcomes ${ }^{(19)}$. There was a significant difference $(p=0.033)$ in endoscopic findings post antifungal therapy between participants who did and did not receive surgery. Ponikau et al. used AMB irrigation for a minimum of three months (range 3-17 months) ${ }^{(18)}$. This significant difference between the regimen duration is a further limitation of this study.

Two further open prospective trials ${ }^{(20,21)}$ failed to report any benefit from topical antifungal agents (Fluconazole and AMB) on endoscopic findings. Both studies lacked controls and randomisation. Despite mixed results, limitations of these open prospective trials include low numbers, high drop-out rates, surgical heterogeneity of participants, variability in regimens and inconsistent outcome measures ${ }^{(20)}$.

\section{Oral antifungals}

From the studies included in this review, only four reported endoscopic outcomes following oral antifungal therapy. Khalil et al. administered oral Itraconazole and topical Fluconazole within one study arm and reported lower disease recurrence rates (endoscopically) ${ }^{(15)}$. The oral Itraconazole group (further study arm) alone did not have reduced recurrence rates, therefore it may be deduced that the beneficial effect was potentially produced by the use of the Fluconazole. Further studies have also reported beneficial outcomes for oral antifungals. Chan et al. (22) prospective pilot study showed an endoscopic improvement (38\% ( $n=12)$ patients) following oral Itraconazole. However, these findings were reported not to be significant as the mean endoscopic stage was the same both pre- and posttreatment. The group studied AFS patients exclusively, however, the numbers were relatively small $(n=32)$. As this study did not have a control group or randomisation, methodological bias would be a major limiting factor.

Sieberling and Wormald also used oral Itraconzaole in AFS patients, but at a lower dose (200mg versus $300 \mathrm{mg}$ daily) ${ }^{(23)}$. Endoscopic findings revealed no recurrence in 44 per cent of participants $(n=23)$. Lee et al. also performed a retrospective chart review on a small number of participants treated with oral Voriconazole $(n=15)$ and oral Posaconazole $(n=5)^{(24)}$. Both treatments were reported to yield improved endoscopic scores. However, dosages of each treatment were not reported within their methodology and endoscopic outcomes were not quantified (i.e. validated scoring system). As both of these studies were retrospective chart reviews, it is difficult to draw any firm conclusions from their results, as the outcomes are likely to be subject to considerable bias within their methodology.

\section{Symptom scores for both topical and oral antifungals} Validated - Patient Reported Outcome Measures (PROM) Data extracted from meta-analysis reported there to be no overall difference between the topical AMB versus the placebo group in terms of PROM ${ }^{(1)}$. The pooled SMD was 0.21 [-0.02 to $0.4495 \% \mathrm{Cl}$, which favoured the control group. However, this study included three trials that actively aimed to exclude AFS ${ }^{(25-}$ ${ }^{27)}$. Liang et al. ${ }^{(16)}$ and Ponikau et al. ${ }^{(17)}$ assessed AFS participants and did not report any statistically significant improvement in RSOM-31(Rhinosinusitis Outcome Measure) and SNOT-20 (Sinonasal Outcome Test) scores, respectively, when comparing their topical AMB treatment groups to their placebos over the full trial periods. Interestingly, Liang et al. did show an initial benefit of AMB at two weeks ( $p=0.018)$, however, this difference was 
not reported at four weeks ${ }^{(16)}$. Data extracted from these two studies for the purpose of the meta-analysis revealed a SMD that favoured the treatment group.

Isaacs et al. showed there was no significant difference between SNOT-20 scores when comparing topical AMB versus controls ( $p$ $=0.33)^{(28)}$. Importantly, it must be noted that two studies from this meta-analysis aimed to exclude AFS patients ${ }^{(26,27)}$ and therefore such results must be interpreted with caution considering their overall outcomes in the context of this review.

Kennedy et al. did not report any benefit using the RSDI (Rhinosinusitis Disability Index) score when comparing oral Terbinafine group to controls ${ }^{(29)}$. Critical appraisal tools identified a low risk of bias from this study.

Chan et al. reported that their RSOM-31 scores showed statistically significant improvement $(p=0.0156)$ following the use of oral Itraconazole ${ }^{(22)}$. Limitations of this open pilot study included a lack of randomisation and the use of controls.

\section{Non-validated symptom scores}

One non-randomised prospective study showed no significant improvement in symptom scores upon AFS and CRS patients following the use of topical AMB ${ }^{(21)}$.

Three studies reported symptomatic improvement, however, were limited by their methodological quality. Ponikau et al. reported that 38/51 participants had improved symptoms following topical AMB in their prospective open study ${ }^{(18)}$. A symptomatic improvement was also shown to occur following the prospective use of topical Fluconazole in 12/16 participants with AFS ${ }^{(20)}$. Finally, the retrospective study by Lee et al. reported that symptoms improved in 80 per cent of participants following oral Voriconazole and oral Posaconazole (24).

Disease specific QOL scores were collated within a meta-analysis (1), which reported no significant improvement in QOL with the use of topical AMB when compared to the placebo. When assessed individually (without the studies which excluded AFS) there was still no benefit reported.

\section{Adverse effects of topical and oral antifungals}

Despite the well documented side effect profile of antifungal agents, no studies have reported any statistically significant adverse effects when compared to control groups. However, individual studies have reported common adverse effects (e.g. hepatic enzyme derangement). Meta-analysis assessing adverse events associated with topical AMB versus a placebo, reported no statistically significant difference between the two groups

(1). A risk ratio for adverse events was calculated at 3.36 [0.86 to $13.0795 \% \mathrm{Cl}]^{(1)}$, thus favouring the controls. The remainder of the individual trials reported adverse events inconsistently and failed to show any statistically significant associations with the therapeutic intervention. Four studies which utilised topical antifungal therapy reported nasal irritation / burning with $A M B$ $(17,18,26,27)$, with one study reported skin itching ${ }^{(16)}$. The use of systemic antifungals were associated with elevation of hepatic enzymes in five studies ${ }^{(15,22,24,30)}$.

\section{Discussion}

It is clear that due to the different quality of studies, inclusion criteria, methodology and treatment regimens comparisons and conclusions are difficult to establish. Within the review, sixteen of the included studies reported objective outcomes in the form of endoscopic findings. The endoscopic findings were either reported as part of validated scoring scales $(1,7,16-19,21,22,25-28)$ or nonvalidated systems ${ }^{(15,20,23,24,30)}$. Seven studies reported endoscopic improvement ${ }^{(15,17-19,22-24)}$, whereas the remainder of the studies reported no benefit (endoscopic) from antifungal agents. When the outcomes were taken into context of the methodology of individual studies, there appears to be no high quality evidence that supports the benefit of topical or oral antifungal agents in improving endoscopic outcomes in patients with AFS.

Patient Reported Outcome Measures (PROM) are important to consider when assessing the effect of an intervention as they relate to the perceived benefit recorded by the patient and therefore reflect upon the quality of life (QOL). Only one study reported statistically significant improvement in PROM following antifungal therapy ${ }^{(22)}$, the remainder of the studies showed no benefit. Therefore, due to a lack of high quality evidence in the literature, this review reports that antifungals do not appear to improve symptoms in patients with AFS.

This review identified that twelve studies assessed the use of topical antifungal therapy in AFS and CRS participants, with only two studies ${ }^{(15,20)}$ exclusively including AFS patients. Therefore, it is clear that there are only very few studies that assess AFS patients alone in the literature. This may be related to the complexity within the diagnosis of the condition and the uncertainty surrounding the disease process, or possibly due to the relatively low incidence in comparison to the other subtypes of CRS. As a result, there proved to be a large degree of heterogeneity in the participant profile within the included studies. It can be argued that this factor may be limited by the reported similarities between the conditions (especially CRS with nasal polyposis and AFS). It must also be noted that it is estimated that 10 per cent of CRS patients carry a diagnosis of AFS. Despite this, it remains to be a challenge to draw accurate conclusions from studies that do not specifically recruit this particular group. Without the presence of large methodologically robust studies that assess AFS participants (through strict diagnostic inclusion criteria), it must be stressed that the current evidence-base must be handled with care.

From analysing the results of this review, it has been identified that a significant influence upon the outcomes of both metaanalysis and the systematic reviews in the literature ${ }^{(1,7,28)}$ are the findings reported by three RCTs of high methodological quality 
(Table 2) ${ }^{(25-27)}$. All of these three studies reported no statistical benefit for the use of topical antifungals in CRS. However, a major limitation with respect to this review relates to their active exclusion of AFS. Therefore, despite the similarities of the disease states (e.g. CRS with polyps and AFS), direct conclusions cannot be drawn from these studies regarding the benefit of antifungals in patients with AFS. In addition to this, overall results of any included meta-analysis ${ }^{(1,28)}$ must be interpreted with caution when considering the AFS patient subgroup alone.

This review included several trials that were found to have varying methodology, which would make interpretation of results and direct comparison difficult. Firstly, it was noted that the duration of treatment significantly differed (range 4 to 68 weeks). As mentioned earlier, Ponikau et al. calculated that a short treatment duration (less than 10 weeks) could be associated with a limited treatment effect ${ }^{(17)}$. Another important study design factor that must be recognised is the mode of application of topical therapy employed in each study. Studies included within this review used topical antifungals in the form of spray, lavage and irrigation. It is believed that nasal lavage may confer a treatment benefit versus other forms of application. Ferguson et al. reported that nasal irrigation in patients with CRS had a therapeutic effect on improving outcomes versus the application of $A M B{ }^{(31)}$. Therefore, outcomes in studies that use irrigation / lavage may be confounded by this effect. Two of the included studies that reported an endoscopic improvement administered their topical therapy by irrigation ${ }^{(18)}$ and lavage ${ }^{(19)}$. In addition to the mode of application, it is also clear that there is a significant degree of variation upon the dosage of topical antifungal delivered between studies included within this review. It has been shown that this may have an effect upon fungal growth. In-vitro studies indicate that fungal growth may not be reduced at concentrations of $\mathrm{AMB}$ at $100 \mu \mathrm{g} / \mathrm{ML}$, whereas impedance is considered to occur at concentrations of $200-300 \mu \mathrm{g} / \mathrm{ML}^{\text {(32) }}$. Helbling et al. used a total daily dose of $3 \mathrm{mg} \mathrm{AMB}$, which was the lowest treatment dose when compared to the other studies included in this review (range $3 \mathrm{mg}$ to $20 \mathrm{mg}$ daily) ${ }^{(21)}$. The group reported no significant benefit of antifungals on endoscopic outcome, which may be partly due to the lower dosage used. The type of topical antifungal studied varied within the literature. $\mathrm{AMB}$ was the most commonly used topical antifungal therapy within the included studies (10 trials), however, topical Fluconazole via a spray or lavage was also studied ${ }^{(15)}$. Bent and Kuhn assessed antifungal activity against AFS organisms during an in vitro study and reported that $A M B$ and Ketoconazole to be the most effective antifungals in vitro ${ }^{(5)}$. It has been reported that Fluconazole has limited antifungal activity in those with AFS. However, in vitro studies have shown mixed results. Current evidence suggests that the type of antifungal agent may also impact on the outcome in AFS, however, further in vivo studies are needed.
Review of the literature for both topical and systemic antifungal therapies identified significant surgical heterogeneity between the studies. Several studies specified within their inclusion criteria for participants to have recently undergone FESS prior to the study ${ }^{(17-20,25)}$ or as part of the study ${ }^{(15,22,23,26,30)}$. However, a number of studies specifically excluded participants whom had recent FESS prior (range 3 to 12 weeks) to the medical intervention ${ }^{(16,21,27,29)}$. The studies that excluded FESS prior to intervention argued that surgery would positively influence outcomes. Several studies have reported that FESS improves the delivery of topical preparations compared to pre-operative states ${ }^{(33,34)}$. This evidence therefore implies that the surgical heterogeneity described within the review may influence outcomes of individual studies especially in the setting of topical application (e.g. spray, irrigation, drops).

\section{Recommendations for future studies}

This review has identified that the current literature has limited high quality evidence that specifically assesses the use of antifungal treatment in patients with AFS. Several factors contributing to the paucity of good quality evidence have been discussed in this review in relation to limitations of existing studies. Therefore, to yield better quality of evidence in the future, trials must be double-blind and randomised which enrol larger numbers of participants who are diagnosed with AFS specifically. It is also important that these participants should be well matched in terms of demographics and surgical state (e.g. previous FESS) to reduce heterogeneity. There should be consideration into the adequate delivery, dosage and duration of antifungal therapies (e.g. sufficient to cause treatment effect). Finally, to obtain consistent objective data for analysis, the use of validated scoring systems (e.g. endoscopic, PROM) must be used within the studies.

\section{Conclusion}

Based upon the findings of this review, there is no convincing evidence to support the routine use of topical or oral antifungals in the treatment of patients with AFS (or CRS). However, it is also clear that there remains to be limited numbers of high quality studies focusing specifically on the use of antifungals in AFS. Therefore, to draw accurate conclusions about the benefit of antifungals within this particular group, recommendations for future research include enrolment of AFS patients into further large multi-centred double-blinded RCTs. These studies should aim to address factors such as the heterogeneity in the surgical state of participants, mode of application, dose and timing of antifungal therapy.

\section{Acknowledgement}

S. Buttress, Academic supervisor MSc Surgical Practice, University of Salford. 


\section{Authorship contribution}

SGM: Researcher, author and BNK: Supervisor, editor.

\section{Conflict of interest}

None.

\section{References}

1. Sacks P, Harvey R, Rimmer J, Gallagher R \& Sacks R. Antifungal therapy in the treatment of chronic rhinosinusitis: a meta-analysis. Am J Rhinol Allergy 2012; 26: 141-147.

2. McClay JE, Meyers AD (editors). Allergic Fungal Sinusitis. Medscape reference (2012) Available from http://emedicine.medscape. com/article/834401-overview [accessed 12 February 2013]

3. Marple B. Allergic Fungal Rhinosinusitis: current theories and management strategies. Laryngoscope 2001; 111: 1006-1019.

4. Thanasumpun T, Batra PS. Oral antifungal therapy for chronic rhinosinusitis and its subtypes: a systematic review. Int Forum Allergy Rhinol. 2011; 1: 382-389.

5. Bent JP, Kuhn FA. Antifungal activity against allergic fungal sinusitis organisms. Laryngoscope 1996; 106: 1331-1334.

6. Ponikau JU, Sherris DA, Kern EB, et al. The diagnosis and incidence of allergic fungal sinusitis. Mayo Clinic Procs 1999; 74: 877884.

7. Sacks P, Harvey R, Rimmer J, Gallagher R, Sacks R. Topical and systemic antifungal therapy for the symptomatic treatment of chronic rhinosinusitis. Cochrane Database Syst Rev. 2011; 8: CD008263.

8. Bent JP, Kuhn FA. The diagnosis of allergic fungal sinusitis. Otolaryngol Head Neck Surg. 1994; 111: 580-588.

9. Fokkens WJ, Lund VJ, Mullol J et al. European Position Paper on Rhinosinusitis and Nasal Polyps 2012. Rhinology. 2012; Suppl. 2012: 1-298.

10. Benninger MS, Ferguson BJ, Hadley JA, et al. Adult chronic rhinosinusitis: definitions, diagnosis, epidemiology and pathophysiology. J Otolaryngol Head Neck Surg. 2003; 123: S1-32.

11. Kupferberg SB, Bent JP, Kuhn FA. Prognosis for allergic fungal sinusitis. J Otolaryngol Head Neck Surg. 1997; 117: 35-41.

12. Lund VJ, Kennedy DW. Staging for rhinosinusitis. J Otolaryngol Head Neck Surg. 1997; 117: S35-40.

13. Malm L. Assessment and staging of nasal polyposis. Acta Oto-laryngologica. 1997; 117(4): 465-7.

14. Centre for Evidence Based Medicine. Oxford centre for evidence based medicine - levels of evidence (2009). Available from http://
www.cebm.net/?O=1025 [accessed 18 Apri 2013]

15. Khalil Y, Tharwat A, Abdou AG, et al. The role of antifungal therapy in the prevention of recurrent allergic fungal rhinosinusitis after functional endoscopic sinus surgery: a randomized, controlled study. Ear Nose Throat J. 2011; 90: E1-7.

16. Liang KL, Su MC, Shiao JY, et al Amphotericin B irrigation for the treatment of chronic rhinosinusitis without nasal polyps: a randomized, placebo-controlled double-blind study. Am J Rhinol. 2008; 22: 52-58.

17. Ponikau JU, Sherris DA, Weaver A, Kita H Treatment of chronic rhinosinusitis with intranasal Amphotericin B: A randomized, placebo-controlled, double-blind pilot trial. J Allergy Clin Immunol. 2005; 115: 125-131.

18. Ponikau JU, Sherris DA, Kita H, Kern EB. Intranasal antifungal treatment in 51 patients with chronic rhinosinusitis. Allergy Clin Immunol. 2002; 110: 862-866.

19. Ricchetti A, Landis BN, Maffioli A, Giger R, Zeng C, Lacroix JS. Effect of anti-fungal nasal lavage with Amphotericin B on nasal polyposis. J Laryngol Otol. 2002; 116: 261 263.

20. Jen A, Kacker A, Huang C, Anand V. Fluconazole nasal spray in the treatment of allergic fungal sinusitis: a pilot study. Ear, Nose, Throat J. 2004; 83: 694-695.

21. Helbling A, Baumann A, Haani C, Caversaccio M. Amphotericin B nasal spray has no effect on nasal polyps. J Laryngol Otol. 2006; 120: 1023-1025.

22. Chan KO, Genoway KA, \& Javer AR. Effectiveness of Itraconazole in the management of refractory allergic fungal rhinosinusitis. J Otolaryngol Head Neck Surg. 2008; 37: 870-874.

23. Sieberling $K$, Wormald $P$. The role of Itraconazole in recalcitrant fungal sinusitis. Am J Rhinol Allergy 2009; 23: 303-306.

24. Lee AS, Przybyszewski B, Montone K, Lanza DC. Oral antifungal therapy for Fusariumassociated chronic rhinosinusitis. Int Forum Allergy Rhinol. 2012; 2: 45-50.

25. Ebbens FA, Scadding GK, Badia L, et al. Amphotericin B nasal lavages: not a solution for patients with chronic rhinosinusitis. J Allergy Clin Immunol 2006; 118: 11491156.
26. Gerlinger I, Fittler A, Fonai F, Patzko A, Mayer A, Botz L. Postoperative application of Amphotericin B nasal spray in chronic rhinosinusitis with nasal polyposis, with a review of the antifungal therapy. Eur Arch Otorhinolaryngol 2009; 266: 847-855.

27. Weschta M, Rimek D, Formanek M, Polzehl D, Podbielski A, Riechelmann H. Topical antifungal treatment of chronic rhinosinusitis with nasal polyps: a randomized, doubleblind clinical trial. J Allergy Clin Immunol 2004; 113: 1122-1128.

28. Isaacs S, Fakhri S, Luong A, Citardi MJ. A meta-analysis of topical Amphotericin B for the treatment of chronic rhinosinusitis. Int Forum Allergy Rhinol. 2011; 1: 250-254.

29. Kennedy DW, Kuhn FA, Hamilos DL, et al. Treatment of chronic rhinosinusitis with high-dose oral terbinafine: a double blind, placebo-controlled study. Laryngoscope 2005; 115: 1793-1799.

30. Rains B, Mineck C. Treatment of allergic fungal sinusitis with high-dose Itraconazole. Am J Rhinol 2003; 17: 1-8.

31. Ferguson B J. Antifungal nasal washes for chronic rhinosinusitis: what's therapeutic - the wash or the antifungal? J Allergy Clin Immunol. 2003; 111: 1137-1138.

32. Shirazi MA, Stankiewica JA, Kammeyer P. Activity of nasal Amphotericin B irrigation against fungal organisms in vitro. Am J Rhinol 2007; 21: 145-148.

33. Grobler A, Weitzel EK, Buele A, et al. Pre- and postoperative sinus penetration of nasal irrigation. Laryngoscope 2008; 118: 2078-2081.

34. Harvey RJ, Goddard JC, Wise SK, Schlosser RJ. Effects of endoscopic sinus surgery and delivery device on cadaver sinus irrigation. J Otolaryngol Head Neck Surg 2008; 139: 137-142.

SG Mistry

Department of Otolaryngology

Bradford Royal Infirmary

Bradford

United Kingdom

Tel: +44-(0)1274-542200

E-mail: smis83@doctors.org.uk 